\title{
Optical Characterization of the Antigen-Antibody Thin Layer Using the Whispering Gallery Mode
}

\author{
Takeshi TaJIRI,***ं Shuzo Matsumoto, ${ }^{*}$ Toshihiko Imato, ${ }^{* * *}$ Toshihiro OKamoto, ${ }^{* *}$ and \\ Masanobu HaRAGUCHI** \\ *Industrial Technology Center of Nagasaki, 2-1303-8 Ikeda, Omura, Nagasaki 856-0026, Japan \\ **Department of Optical Science and Technology, Faculty of Engineering, The University of Tokushima, 2-1 \\ Minamijosanjima, Tokushima 770-8506, Japan \\ ***Department of Applied Chemistry, Graduate School of Engineering, Kyushu University, 744 Moto-oka, \\ Nishi, Fukuoka 819-0395, Japan
}

\begin{abstract}
We immobilized an antibody (anti- $\beta$-Galactosidase) on a polystyrene microsphere by using a covalent bond, and observed the resonance peaks in the scattered light intensity spectra related to the whispering gallery mode (WGM) excitation of the microsphere. The amount and the optical parameters, i.e., thickness and refractive index, of anti- $\beta$-Galactosidase on the sphere surface were evaluated based on an absorbance measurement and a resonance peak shift measurement, respectively. Moreover, we measured the variation of the WGM spectra depending on the concentration of the enzyme solution ( $\beta$-Galactosidase), which allowed us to optically evaluate the thickness and the refractive index of the antigen-antibody layer from the shift of the WGM spectra peak.
\end{abstract}

Keywords Microsphere, whispering gallery mode, resonance sensors, biosensors

(Received May 21, 2014; Accepted June 9, 2014; Published August 10, 2014)

\section{Introduction}

As interest of food safety relief rises, a voluntary laboratory procedure to prevent microbial contamination is necessary. Because the conventional method requires a long inspection time, we often obtain the test result after food product shipment. Therefore, we need a quick test to check food safety. However, the current tester has problems, e.g. quick aspect, handiness, precision and inspection cost, to satisfy such demands. Recently, for the label-free optical detection of viruses and pathogens, ${ }^{1-7}$ whispering gallery mode (WGM) resonators with a high $\mathrm{Q}$ factor have attracted attention. Because the WGM of a microsphere is sensitive to the change of the refractive index of the microsphere environment, the WGM microsphere resonator may work as an optical biosensor with high sensitivity and a short measurement time. ${ }^{8-11}$ Some research groups have successfully excited the WGM in microspheres through the coupling of evanescent light. ${ }^{12-26}$ In particular, the attenuatedtotal-reflection (ATR) geometry has an advantage of an easy sample setting. Using the ATR configuration, we have already reported on the observation of resonance signals related to WGMs in microspheres with $\mathrm{TiO}_{2}$ thin film, and evaluated the optical properties of the film on the microsphere by using the WGM resonance. ${ }^{27}$

However, it is difficult for a single microsphere sensor to estimate the state of the immobilized antibody on a microsphere surface. There is a method used to evaluate the state, where the antibody labeled a fluorescent dye undergoes fluorescence by the antigen-antibody reaction. However, the inspection processes increase, and the cost of the sensor tip becomes higher. ${ }^{28-30}$ In addition, because a microsphere becomes a multilayered structure, any optical analysis and evaluation are complicated, and the $S / N$ ratio of the detection light may decrease. Therefore, the manufacturing a single microsphere probe and the establishment of a method for measurement to detect scattered light corresponding to WGM excitation are necessary for the development of biosensors.

In this paper, we report on the observation of discrete changes in the wavelength of WGMs as microbial contamination bind to a microsphere cavity. We immobilized an antibody (anti- $\beta$ Galactosidase) of an enzyme ( $\beta$-Galactosidase) on a polystyrene (PS) microsphere. $\beta$-Galactosidase is a production enzyme of the coliform bacteria, which is a hygiene index of food. The binding of anti- $\beta$-Galactosidase on the microsphere surface was evaluated by absorbance measurements at the ultraviolet wavelength of $\lambda=280 \mathrm{~nm}$. Furthermore, the manufactured single microsphere was excited at the total internal reflectionexcitation configuration by a white light using an immersion object lens $(100 \times)$, and detected the emission spectra corresponding to WGM. As a result of having compared the experimentally obtained spectra with those obtained from Mie scattering theory, it was characterized that a surface state of the microsphere was reflected by the WGM spectra.

$\dagger$ To whom correspondence should be addressed.

E-mail: tajiri@tc.nagasaki.go.jp 
(2); Coupling solution

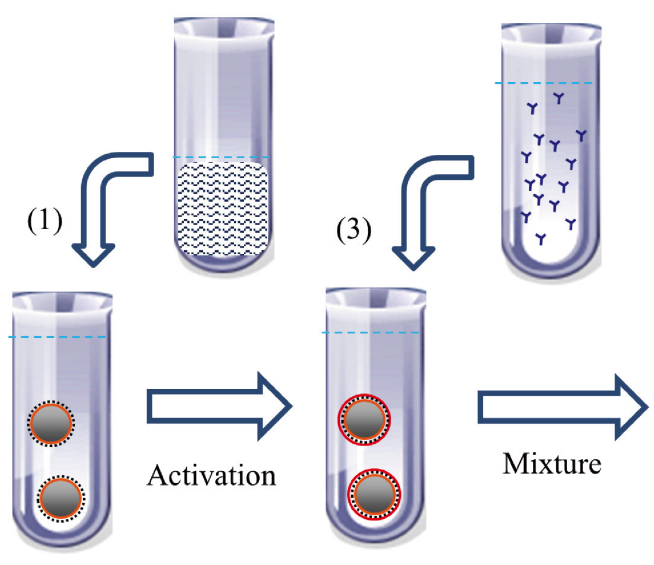

(4); Start solution of the antibody

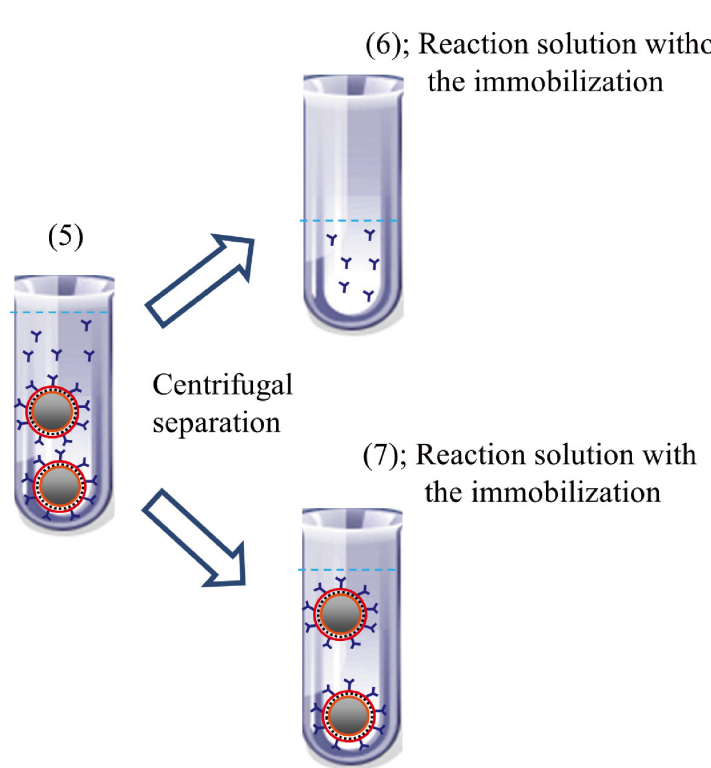

Fig. 1 Schematic diagram of the fabrication process to immobilize the antibody (anti- $\beta$-Galactosidase) on PS microspheres. (1) dispersion of a PS microsphere of the carboxyl group (COOH) modification in a water solution; (2) carbodiimide solution to employ as a coupling solution; (3) activation of the carboxyl group ( $\mathrm{COOH}$ ) on the PS microsphere; (4) antibody (anti- $\beta$-Galactosidase) solution before reacting to a PS microsphere; (5) mixed solution of the PS microsphere and the antibody; (6) antibody solution which was not immobilized to PS microsphere; (7) solution which has an antibody immobilized to a PS microsphere.

\section{Experimental}

\section{Reagents and chemicals}

A PS microsphere was purchased from micromod Partikeltechnologie GmbH (Germany, \#01-02-104). The diameter of the PS microsphere size was $10 \mu \mathrm{m}(\mathrm{CV}$ value < $10 \%)$, and a carboxyl group $(\mathrm{COOH})$ was modified. An antibody (anti- $\beta$-Galactosidase, from Rabbit $\mathrm{IgG}$ ) bound to the PS microsphere was purchased from Medical \& Biological Laboratories Co., Ltd. (Japan). The chemicals for bonding of the PS microsphere and an antibody was purchased from Polysciences Inc. (USA, Polylink Protein Coupling Kit). The enzyme ( $\beta$-D-Galactosidase, from Escherichia coli) of the antigen-antibody reaction was purchased from Wako Pure Chemical Industries, Ltd. (Japan). Ultra-pure water was prepared by a Milli-Q system and used in all experiments.

Immobilization and evaluation method of anti- $\beta$-Galactosidase

We have to employ a microsphere with high sphericity surface and a high refractive index in order to support WGM resonance..$^{27,30}$ Here, a PS microsphere (diameter of $10 \mu \mathrm{m}$ ) having a modified carboxyl group $(\mathrm{COOH})$ was employed. Figure 1 shows the growth process for immobilizing an antibody (anti- $\beta$-Galactosidase) on a single dispersion PS microsphere. After having washed several times, the PS microsphere mixes with the coupling solution of the water-soluble carbodiimide. After being incubated for one hour at room temperature with gentle mixing, the carboxyl group modified by the microsphere surface is activated by water-soluble carbodiimide, and covalently binds by reacting with an amine of the antibody (anti- $\beta$-Galactosidase). The mixture of the microsphere including the antibody is centrifuged, and the antibody without immobilization is removed. We repeated these washing and centrifugal separation steps several times to obtain a microsphere where an immobilized antibody.

The amount of binding of the antibody (anti- $\beta$-Galactosidase) on the microsphere surface was estimated by comparing the reaction solution (Fig. 1(6)) with the start solution (Fig. 1(4)). The separation solution was diluted to 15 times, and was measured by ultraviolet wavelength $(280 \mathrm{~nm})$ light of the spectrophotometer (Shimadzu Corp., UV-1200). Because the absorbance measurement employed a 15-times dilution solution, in fact, the amount of the antibody of 15 times immobilized to the surface of the PS microsphere.

\section{Optical measurements system and evaluation method}

A white light source (Energetiq Technology Inc. (USA), LDLS-EQ99, $170-2100 \mathrm{~nm}$ ) was employed for the excitation light of the PS microsphere. The PS microsphere was placed on a borosilicate glass base dish (Asahi Glass Co., Ltd., base glass diameter $12 \mathrm{~mm}$, base glass thickness $0.15-0.18 \mathrm{~mm}$ ). Moreover, the cover glass (Matsunami Glass Ind., Ltd., $24 \times$ $24 \times 0.12-0.17 \mathrm{~mm}$ ) was covered for preventing any drying of the solution. A single PS microsphere was excited at the total internal reflection-excitation configuration by the immersion object lens (Nikon Corp., CFI 100× H, NA 1.25), and detected spectra corresponding to WGM. This configuration is essentially the same as the ATR experimental set-up proposed by Ishikawa et al. $^{31}$

The experiment system and the excited state to the PS microsphere is shown in Figs. 2(a) and 2(b). As shown in Fig. 2(a), a probe light beam was irradiated in the microsphere from the glass side using an immersion objective lens. The excitation position to the PS microsphere was adjusted by an $\mathrm{XYZ}$ translation stage. Moreover, as shown in the CCD image 
(a)

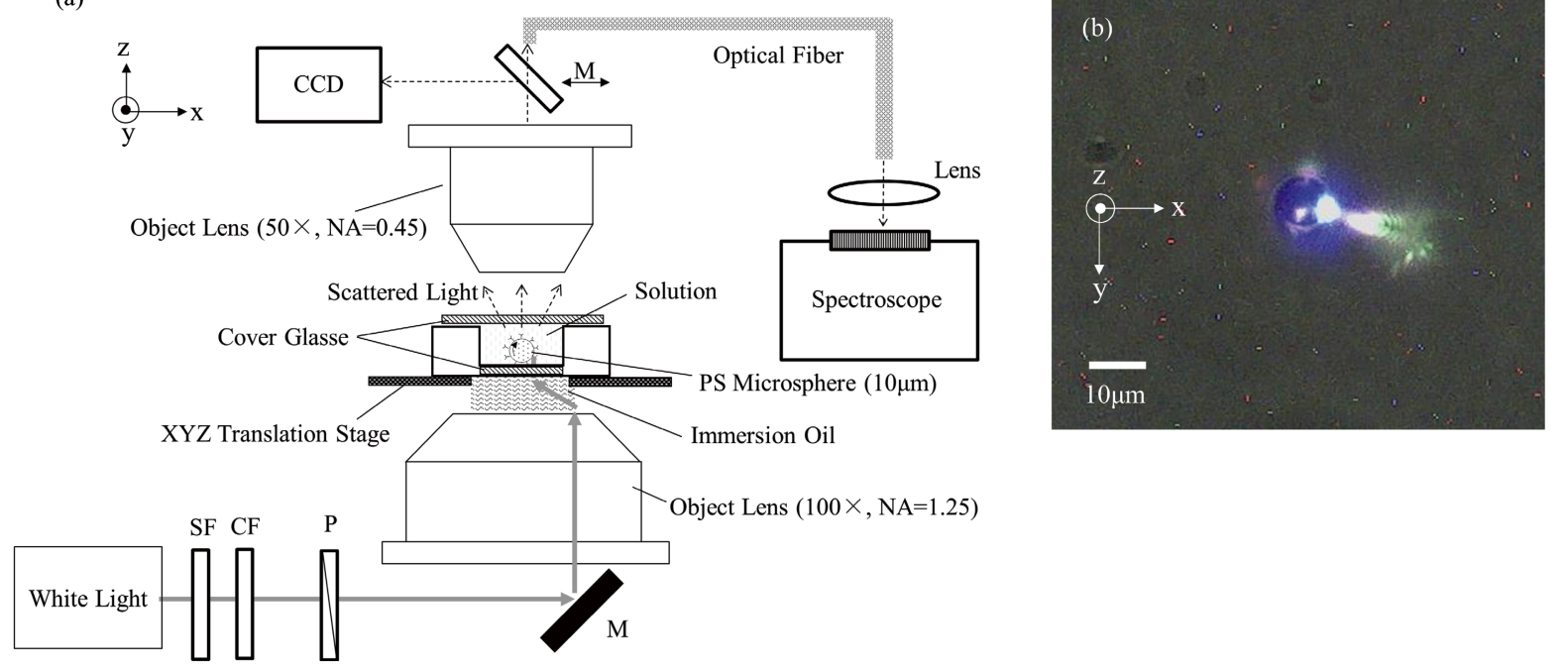

Fig. 2 Optical measurements system of the microsphere. (a) Experimental setup for WGM spectroscopy; SF, sharp cut filter; CF, cold filter; P, polarizer; M, plane mirror. (b) CCD image of PS microsphere excitation.

of Fig. 2(b), the excitation light was focused to form a smaller spot than the diameter of the PS microsphere. Therefore, because the scattered light from contaminants in the substrate surface and the solution was decreased, most of the signal only from single microsphere was detected, and the $S / N$ ratio was increased. As shown in Fig. S1 (Supporting Information), because the center position of the PS microsphere contacts with the cover glass, the evanescent light that seeped to inside of the PS microsphere was scattered as normal transmitted light without propagating to the surface. Therefore, in an excitation position of the PS microsphere, the end face position was chosen. The PS microsphere was excited only by the $X$ direction, and the scattered light that went around the $\mathrm{XZ}$ plane was detected. The WGM in the PS microsphere was excited by TE or TM polarized light obtained by the combination a white light source and a polarizer (Sigma Koki Co., Ltd., GTPC-10$25 \mathrm{AN})$. The incident angle of the excitation light was adjusted more greatly than 50 degrees, which is larger than the critical angle for total internal reflection at the glass-air interface. Ultraviolet and the infrared excitation light were interrupted by a sharp-cut filter and cold filter (Sigma Koki Co., Ltd., SCF50S-37L and CLDF-50S). The scattered light from PS microsphere was focused by an object lens (Nikon Corp., CF IC EPI Plan SLWD 50× A), and detected with a spectroscope (Hamamatsu Photonics K.K., PMA50).

Any variation of the WGM resonance was inspected by detecting scattered light of the PS microsphere, which varied in a surface condition. In order to explore the effect of the PS microsphere surface on the resonance, we calculated the theoretical scattering cross-section spectra of a PS microsphere without and with a coated antigen-antibody reaction layer using Mie scattering theory. The scattering cross-section was calculated numerically by the program using Mie scattering theory. ${ }^{32-34}$ In addition, to examine the thickness and the density state of the immobilized antigen antibody on PS microsphere, the time-course of WGM after having dropped an enzyme solution ( $\beta$-Galactosidase) was examined. The graph fitting of the time-course employed the Origin Version 8.1 software (LightStone Corp.). Moreover, we measured any variation of the WGM spectra depending on the concentration of the enzyme
( $\beta$-Galactosidase). The concentration of the enzyme ( $\beta$-Galactosidase) was varied from 1 to $1000 \mu \mathrm{g} / \mathrm{mL}$.

\section{Results and Discussion}

\section{Evaluation of the antibody immobilization by absorbance measurement}

The binding of anti- $\beta$-Galactosidase on the microsphere surface was evaluated by absorbance comparisons between various samples at an ultraviolet wavelength of $\lambda=280 \mathrm{~nm}$. Here, the absorbance at an ultraviolet wavelength of $\lambda=280 \mathrm{~nm}$ was shown with $\mathrm{A}_{280}$. The compared absorbance is solution (4) and (6) of Fig. 1, respectively. The absorbance of the start solution of the antibody was $\mathrm{A}_{280}=0.136$, and the absorbance of reaction solution without the immobilization after one hour was $\mathrm{A}_{280}=0.093$. The coupling reagent of the carbodiimide was contained in solution (6) of Fig. 1, but the absorbance of the carbodiimide is very feeble with $\mathrm{A}_{280}=0.005$. Therefore, the carbodiimide does not contribute to any absorbance change, and any absorbance change means that an antibody bound to the PS microsphere surface. The absorbance at $\lambda=280 \mathrm{~nm} \mathrm{~A} \mathrm{~m}_{280}$ and the protein concentration are expressed by the following relations (1):

$$
\mathrm{A}_{280}=1.0=1.0 \mathrm{mg} / \mathrm{mL}=1.0 \mu \mathrm{g} / \mu \mathrm{L} .
$$

Because the absorption degree measurement employed by 15-times dilution, in fact, the amount of antibody of 15 times immobilizes on the PS microsphere surface. The concentration of the antibody calculated by absorbance of the solution at the start and the reaction were 2.040 and $1.395 \mu \mathrm{g} / \mu \mathrm{L}$, respectively. Therefore, by the subtraction of the start solution and the reaction solution, the antibody concentration of $0.645 \mu \mathrm{g} / \mu \mathrm{L}$ was bound to a microsphere. The ratio of the amount of antibody immobilized on the PS microsphere surface was more than $30 \%$ compared to that of the start solution. By the commercial specifications of PS microspheres employed here, the PS number of particles is $9.3 \times 10^{7}$ per $\mathrm{mL}$. Therefore, the amount of the immobilized antibody was estimated to be 

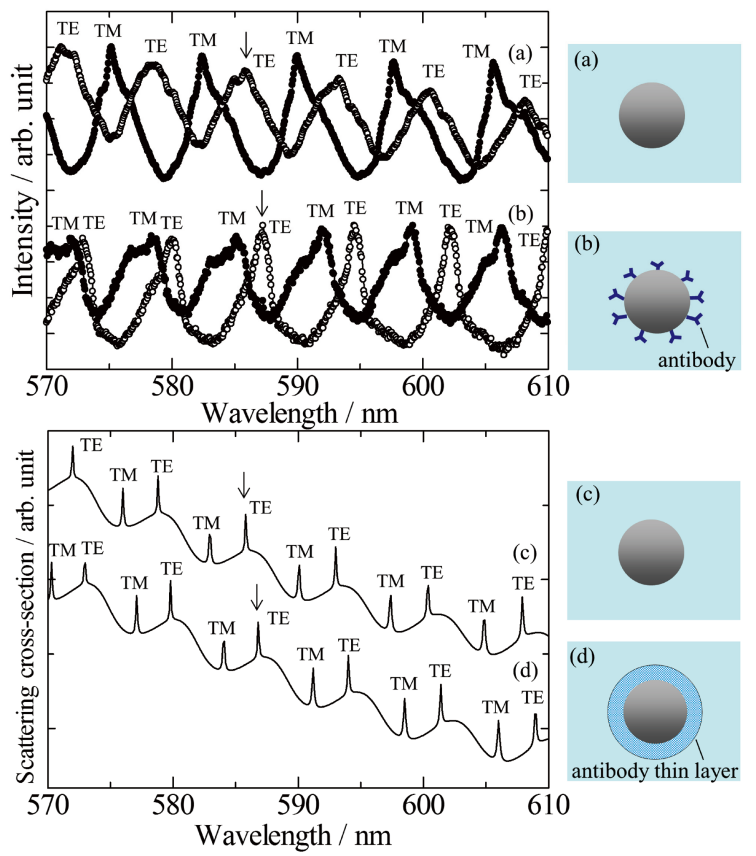

Fig. 3 Here, (a) and (b) are the scattered light intensity spectra of the PS microsphere without and with the antibody immobilized. The solid (๑) and open $(\bigcirc)$ circles are for the TM- and TE-polarized light, respectively; (c) and (d) are theoretical scattering cross-section spectra of a PS microsphere without and with the antigen-antibody thin layer using Mie scattering theory. The arrow indicates the shift of the spectra due to the TE polarization.

\section{$6.935 \mathrm{pg}$ per single microsphere.}

WGM spectra evaluation of the PS microsphere in water

The WGM spectra was detected in order to inspect the conditions of the PS microsphere surface. Figures 3(a) and 3(b) show the scattered light intensity spectra of the PS microsphere without and with the antibody immobilized, respectively. The PS microsphere was placed on a cover glass in water. The solid and open circles of Figs. 3(a) and 3(b) are for the TM- and TE-polarized light, respectively. Because the WGM resonance peak shift is not influenced by the scattered light intensity, the scattered intensities are normalized by the maximum scattered intensity. In Figs. 3(a) and 3(b), there are 5 to 6 peaks where an interval is about $7 \mathrm{~nm}$. These periodic peaks are thought to be related to the excitation of the WGMs in the microsphere. ${ }^{12,35,36}$

Figures 3(c) and 3(d) show the theoretical scattering cross-section spectra of a PS microsphere without and with the antigen-antibody thin layer using Mie scattering theory. Note that the substrate is not considered in the calculations, and that the incident light is a polarized plane wave. From Mie scattering theory, the scattered intensity peaks correspond to the excitation wavelengths of the WGMs. In the calculations of Fig. 3(c), the diameter of the PS microsphere was set to $10.04 \mu \mathrm{m}$. This value almost agrees with the commercial specifications of the PS microspheres employed here. The refractive index of the microsphere and surroundings water were set to 1.59 and 1.33 , respectively. Therefore, the carboxyl group $(\mathrm{COOH})$ did not contribute to WGM spectra change.

WGM spectra evaluation of the antibody immobilization PS microsphere in water

As shown in Fig. 3(b), the scattered light peaks interval of the

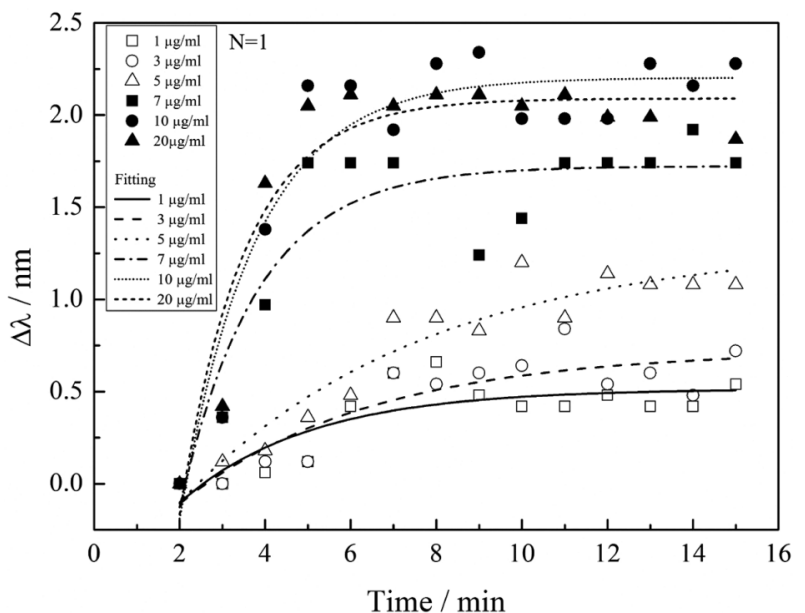

Fig. 4 TE-WGM shift $(\Delta \lambda)$ of the time-course by the antigenantibody reaction upon the same microsphere. The plots are experimental data, and the lines are the result by the best-fit using exponential trendline.

immobilized microsphere are the almost same as those of Fig. 3(a). On the other hand, compared with the spectra presented in Fig. 3(a), there are differences in the wavelength positions of the peak in Fig. 3(b). The peak wavelength of the immobilized microsphere shifts to about $1 \mathrm{~nm}$ toward longer wavelength than that of a microsphere without immobilization. The resonance frequencies of the WGMs are sensitive to the diameter and the refractive-index of the microsphere. Therefore, the existance of the immobilized antibody leads to wavelength shifts. ${ }^{6,37,38}$

Moreover, as shown in Fig. 3(d), in order to explore the effect of an antibody thin layer on the resonance, we calculated the theoretical scattering cross-section spectra of a PS microsphere using Mie scattering theory. As shown in Figs. 3(b) and 3(d), the experimental scattered peaks of TE- and TM-WGMs almost agree with the theoretical scattering cross-section spectra. In the calculations, the diameter of the PS microsphere was set to $10.04 \mu \mathrm{m}$, and the refractive index of the microsphere and surrounding solution were set to 1.59 and 1.33 , respectively. The refractive index, $\mathrm{n}_{\mathrm{c}}$, and the thickness, $\mathrm{t}$, of the immobilized antibody were estimated to be 1.50 and $14 \mathrm{~nm}$, respectively. The values of the refractive index of the antibody agrees with the refractive index of the protein by Fan et al., ${ }^{5}$ i.e., 1.5, and that by Voros, ${ }^{39}$ i.e., $1.35-1.6$. Furthermore, the size of the immobilized antibody is similar to that of the "Y" model size of the IgG antibody (Peace III et al. and Tan et al.). ${ }^{40,41}$ Therefore, we conclude that antibody "Y" immobilized on the PS microsphere surface is a monolayer.

\section{Characterization of the antigen-antibody reaction layer}

In order to discuss a state on the PS microsphere surface based on an antigen-antibody reaction, we obtained the variation of the WGM spectra depending on the concentration of the enzyme solution ( $\beta$-Galactosidase). From the reaction kinetics of the antigen-antibody reaction, the complicated concentration of the antigen antibody increases by time-course exponentially. ${ }^{42}$ Therefore, as shown in Fig. 4, the TE-WGM resonance peaks shift $(\Delta \lambda)$ by antigen-antibody reaction was detected in order to examine the time-course of the antigen antibody upon the same microsphere. In this experiment, because the microsphere in a reaction solution was excited after cover glass sealing, the 


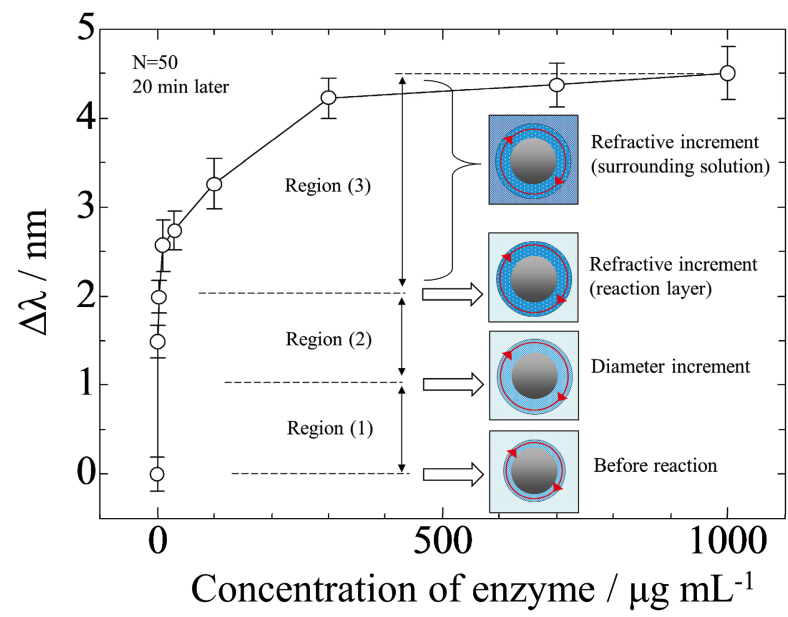

Fig. 5 TE-WGM resonance peaks shift $(\Delta \lambda)$ by the increment of enzyme concentration. $\Delta \lambda$ is the average value of the microsphere $(\mathrm{N}=50)$, and shows $20 \mathrm{~min}$ after enzyme dropping. The model shows the reaction of antigen antibodies from region (1) to (3).

detection of the TE-WGM resonance peak shift $(\Delta \lambda)$ was started approximately $2 \mathrm{~min}$ after the enzyme ( $\beta$-Galactosidase) was dropped. The TE-WGM resonance peaks shift $(\Delta \lambda)$ increased with the increment of the reaction time exponentially, and a high concentration tended to be quick in the antigen-antibody reaction. Afterwards, the TE-WGM resonance peaks shift $(\Delta \lambda)$ were saturated by a concentration of $10 \mu \mathrm{g} / \mathrm{mL}$, and tended to level off after $8 \mathrm{~min}$.

Figure 5 shows the TE-WGM resonance peaks shift $(\Delta \lambda)$ by the increment of the enzyme concentration. $\Delta \lambda$ shows the increment from the arrow at $\lambda=586 \mathrm{~nm}$ of Fig. 3(b) of $20 \mathrm{~min}$ after enzyme dropping. There is a nonuniformity of the immobilization to the microsphere, respectively; therefore, the shift amount $(\Delta \lambda)$ shows the average of microsphere $(\mathrm{N}=50)$. The enzyme concentration was increased from 1 to $1000 \mu \mathrm{g} / \mathrm{mL}$, and the WGM resonance peaks were shifted to long wavelength at a maximum of $\Delta \lambda=4.5 \mathrm{~nm}$. As shown in Fig. 5, the increment of $\Delta \lambda$ tends to be rapid in regions (1) and (2), and levels off in regions (3). Because the covered rate of the microsphere goes up when enzyme concentration increases, the resonance wavelength becomes longer.

Compared with the $\Delta \lambda$ presented in Figs. 4 and 5, respectively, regions (1) and (2) and (3) in Fig. 5 can be explained as follows. Region (1): The reaction layer thickness on the microsphere surface increases due to the antigen-antibody reaction. Because accumulation of the antigen-antibody layer increases the size of the polystyrene microsphere, the WGM resonance shifts to a longer wavelength. Region (2): At first, in accordance with any enzyme concentration increases, the density of the antigenantibody reaction layer is increased on the microsphere surface; as a result, the coated refractive indexes increase. Region (3): Thereafter, the enzyme without immobilizing increases the concentration of the microsphere surroundings; as a result, the refractive indexes of the surrounding solution increase.

As shown in Table 1, we calculated the best-fit theoretical spectra of the scattering cross-section of a PS sphere to the experimental spectra by using Mie scattering theory, in order to evaluate the thickness and the refractive index of the antigenantibody reaction layer. In the calculations, the diameter of the PS microsphere was set to $10.04 \mu \mathrm{m}$, and the refractive index of the microsphere was set to 1.59. As a result of fitting in Region
Table 1 Calculation of the resonance peak shift and the reaction layer using the Mie scattering theory

\begin{tabular}{ccccc}
\hline $\begin{array}{c}\text { Resonance } \\
\text { peak } \\
\begin{array}{c}\text { avelength } \\
(\lambda) / \mathrm{nm}\end{array}\end{array}$ & $\begin{array}{c}\text { Resonance } \\
\text { peaks } \\
\text { shift } \\
(\Delta \lambda) / \mathrm{nm}\end{array}$ & $\begin{array}{c}\text { Reaction } \\
\text { layer } \\
\text { thickness } \\
(\mathrm{t}) / \mathrm{nm}\end{array}$ & $\begin{array}{c}\text { Refractive } \\
\text { index of a } \\
\text { reaction layer } \\
\left(\mathrm{n}_{\mathrm{c}}\right)\end{array}$ & $\begin{array}{c}\text { Refractive } \\
\text { index of } \\
\text { surrounding } \\
\text { solution }\left(\mathrm{n}_{\mathrm{s}}\right)\end{array}$ \\
\hline 585.8 & -1.0 & 0 & 0 & 1.33 \\
586.9 & 0 & 14 & 1.50 & 1.33 \\
587.9 & 1.1 & 30 & 1.50 & 1.33 \\
588.3 & 1.5 & 30 & 1.53 & 1.33 \\
588.8 & 2.0 & 30 & 1.56 & 1.33 \\
589.6 & 2.8 & 30 & 1.56 & 1.35 \\
590.1 & 3.3 & 30 & 1.56 & 1.36 \\
590.6 & 3.8 & 30 & 1.56 & 1.37 \\
591.1 & 4.3 & 30 & 1.56 & 1.38 \\
\hline
\end{tabular}

In the calculations of TE-WGM, the diameter of the PS microsphere was set to $10.04 \mu \mathrm{m}$, and the refractive index of the microsphere was set to 1.59. In an antigen-antibody reaction layer, the refractive indexes of an antibody and the enzyme were set to the same value.

(1), the thickness, $t$, of the antigen-antibody reaction layer and the refractive index, $n_{c}$, of the reaction layer and the refractive index, $\mathrm{n}_{\mathrm{s}}$, of the surrounding solution were estimated to be $30 \mathrm{~nm}, 1.50$ and 1.33 , respectively. Here, the refractive indexes of an antibody and the enzyme are set to the same value. The thickness $(30 \mathrm{~nm})$ of the antigen-antibody layer calculated from a fitting contains an antibody (anti- $\beta$-Galactosidase) of $14 \mathrm{~nm}$. Therefore, the thickness of the enzyme ( $\beta$-Galactosidase) is calculated with $16 \mathrm{~nm}$; this value agrees with the diameter of $\beta$-Galactosidase that Skalova et al. reported. ${ }^{43}$ In the fitting of Region (2), only the refractive index, $\mathrm{n}_{\mathrm{c}}$, of the reaction layer changed to $1.50-1.56$. The refractive index of the antibody by this work agrees with the refractive index of the protein by Fan et al., ${ }^{5}$ i.e., 1.5, and that by Voros, ${ }^{39}$ i.e., 1.35 - 1.6. In region (3), only the refractive index, $n_{s}$, of the surrounding solution changed to $1.33-1.38$. Maier et al. reported that the refractive index of the glucose mixed solution was proportional to the increase of the glucose concentration. ${ }^{44}$ These relations mean that the solution refractive index was increased by the enzyme concentration. Moreover, Francois and Himmelhaus estimated the refractive index variation $(1.33-1.36)$ of the mixed solution (water and glycerol) surrounding the microsphere by changing concentration of glycerol (refractive index $\approx 1.47$ ). ${ }^{9} \quad$ These refractive index variations are similar to the variation that mixed the enzyme (refractive index $\approx 1.50$ ) by this work.

\section{Conclusions}

We immobilized an antibody ( $\beta$-Galactosidase) on a PS microsphere by using a covalent bond. The immobilized quantity of the antibody was measured based on the absorbance. The results show that an antibody immobilizes more than $30 \%$. From the observation of the WGM resonance peak shift of the microsphere, we were able to optically evaluate the thickness and the refractive index of the antigen-antibody layer on the microsphere by comparing the experimentally obtained spectra with those obtained from Mie scattering theory. Its thickness and refractive index were determined to be $30 \mathrm{~nm}$ and $1.50-1.56$, respectively. An antibody and the enzyme reacted by $1: 1$, and the thickness of an antibody and the enzyme were 14 and $16 \mathrm{~nm}$, respectively. Our developed measurement 
technique can be applied to the bond evaluation of a microsphere and the antibody and antigen. Furthermore, by detecting a resonance peak shift, the judgement of about $10 \mathrm{~min}$ is possible. This suggests a possibility to develop the biosensor to provide rapid detection.

\section{Supporting Information}

This material is available free of charge on the Web at http:// www.jsac.or.jp/analsci/.

\section{References}

1. H. C. Ren, F. Vollmer, S. Arnold, and A. Libchaber, Opt. Express, 2007, 15, 17410.

2. N. M. Hanumegowda, I. M. White, H. Oveys, and X. Fan, Sens. Lett., 2005, 3, 1.

3. M. A. Cooper, Anal. Bioanal. Chem., 2003, 377, 834.

4. A. M. Armani, R. P. Kulkarni, S. E. Fraser, R. C. Flagan, and K. J. Vahala, Science, 2007, 317, 783.

5. X. Fan, I. M. White, S. I. Shopova, H. Zhu, J. D. Suter, and Y. Sun, Anal. Chim. Acta, 2008, 620, 8.

6. F. Vollmer and S. Arnold, Nat. Methods, 2008, 5, 591.

7. S. Arnold, S. I. Shopova, and S. Holler, Opt. Express, 2010, $18,281$.

8. H. Zhu, I. M. White, J. D. Suter, P. Dale, and X. Fan, Opt. Express, 2007, 15, 9139.

9. A. Francois and M. Himmelhaus, Sensors, 2009, 9, 6836.

10. I. Teraoka, S. Arnold, and F. Vollmer, J. Opt. Soc. Am. B, 2003, 20, 1937.

11. K. R. Hiremath and V. N. Astratov, Opt. Express, 2008, 16, 5421.

12. M. L. Gorodetsky and V. S. Ilchenko, Opt. Commun., 1994, $113,133$.

13. A. Serpenguzel, S. Arnold, and G. Griffel, Opt. Lett., 1995, 20,654

14. N. Debreuil, J. C. Knight, D. Leventhal, V. sandoghdar, J. Hare, and V. Lefevere-Seguin, Opt. Lett., 1995, 20, 813.

15. J. C. Knight, G. Cheung, F. Jacques, and T. A. Birks, Opt. Lett., 1997, 22, 1129.

16. V. S. Ilchenko, X. S. Yao, and L. Maleki, Opt. Lett., 1999, 24, 723 .

17. M. Noto, D. Keng, I. Teraoka, and S. Arnold, Biophys. J., 2007, 92, 4466.

18. F. Vollmer, S. Arnold, D. Braun, I. Teraoka, and A. Libchaber, Biophys. J., 2003, 85, 1974.

19. M. A. S. Cordoba, S. V. Boriskina, F. Vollmer, and M. C. Demirel, Appl. Phys. Lett., 2011, 99, 073701.
20. F. Vollmer, D. Braun, A. Libchaber, M. Khoshsima, I. Teraoka, and S. Arnold, Appl. Phys. Lett., 2002, 80, 4057.

21. D. Keng, S. R. McAnanama, I. Teraoka, and S. Arnold, Appl. Phys. Lett., 2007, 91, 103902.

22. I. M. White, N. M. Hanumegowda, and X. Fan, Opt. Lett., 2005, 30, 3189.

23. S. Arnold, M. Khoshsima, I. Teraoka, S. Holler, and F. Vollmer, Opt. Lett., 2003, 28, 272.

24. S. Arnold, D. Keng, S. I. Shopova, S. Holler, W. Zurawsky, and F. Vollmer, Opt. Express, 2009, 17, 6230.

25. T. Ioppolo, N. Das, and M. V. Otugen, J. Appl. Phys., 2010, 107, 103105.

26. A. T. Rosenberger, Opt. Express, 2007, 15, 12959.

27. M. Haraguchi, F. Komatsu, K. Tajiri, T. Okamoto, M. Fukui, and S. Kato, Surf. Sci., 2004, 548, 59.

28. N. Soh, M. Tanaka, K. Hirakawa, R. Zhang, H. Nakajima, K. Nakano, and T. Imato, Anal. Sci., 2011, 27, 1069.

29. H. Kuramitz, Y. Mawatari, M. Ikeuchi, O. Kutomi, N. Hata, S. Taguchi, and K. Sugawara, Anal. Sci., 2012, 28, 77.

30. Y. Tsuboi, R. Shimizu, T. Shoji, and N. Kitamura, Anal. Sci., 2010, 26, 1241.

31. H. Ishikawa, H. Tamaru, and K. Miyano, Opt. Lett., 1999, $24,643$.

32. J. A. Stratton, "Electromagnetic Theory", 1941, McGrawHill, New York.

33. P. W. Barber, and S. C. Hill, "Light scattering by particles: computational methods", 1990, World Scientific, Singapore.

34. M. Fukui and M. Ohtsu, "Hikari Nanotechnology no Kiso (Fundamentals of optical nanotechnology, in Japanese)", 2003, Ohmsha, Ltd., Tokyo.

35. A. Shinya and M. Fukui, Opt. Rev., 1999, 6, 215.

36. M. Haraguchi, T. Nakai, A. Shinya, T. Okamoto, M. Fukui, T. Koda, R. Shimada, and K. Takeda, Opt. Rev., 1999, 6, 261.

37. M. Himmelhaus, S. Krishnamoorthy, and A. Francois, Sensors, 2010, 10, 6257.

38. Y. Sun and X. Fan, Anal. Bioanal. Chem., 2011, 399, 205.

39. J. Voros, Biophys. J., 2004, 87, 553.

40. L. F. Pease III, J. T. Elliott, D.Tsai, M. R. Zachariah, and M. J. Tarlov, Biotechnol. Bioeng., 2008, 101, 1214.

41. Y. H. Tan, M. Liu, B. Nolting, J. G. Go, J. G. Hague, and G. Y. Liu, ACS Nano, 2008, 2, 2374.

42. Y. Katakura, "Baiopurocesu sisutemu (Bioprocess System, in Japanese)", ed. H. Shimizu, 2009, CMC Publishing Co., Ltd., Tokyo, 79.

43. T. Skalova, J. Dohnalek, V. Spiwok, P. Lipovova, E. Vondrackova, H. Petrokova, J. Duskova, H. Strnad, B. Kralova, and J. Hasek, J. Mol. Biol., 2005, 353, 282.

44. J. S. Maier, S. A. Walker, S. Fantini, M. A. Franceschini, and E. Gratton, Opt. Lett., 1994, 19, 2062. 